\title{
Deterministic Computer Simulations of Grazing Impacts on Planetary Surfaces
}

\author{
C.J. Massina ${ }^{\ddagger}$ and M. W. Roth \\ Department of Physics \\ University of Northern lowa \\ Cedar Falls, lowa 50614-0150 USA \\ Paul A. Gray
Department of Computer Science
University of Northern lowa
Cedar Falls, lowa 50614-0507 USA
}

Received: October 29, 2008

Accepted: January 23, 2009

\begin{abstract}
Many bodies in the solar system have features which could conceivably have been formed by a grazing impact with a comet or asteroid. We present the results of deterministic computer simulations of various objects striking a terrestrial planet at a grazing angle. The system is modeled using a combination of the Material Point Method (MPM) and classical planetary dynamics. The impact exhibits three distinct regimes: $(i)$ the initial stage where rapid ejecta leaves the planet in a nearly straight line, (ii) the intermediate stage where the ejecta begins to curve in towards the planet and the trench is being created on the surface and the (iii) the long term stage where the trench is created and any paths exhibited by the ejecta are stable capture orbits. In the case of Mars, we show that a grazing impact can not only dig a trench which has the same general morphology as Valles Marineris but also can create ejecta which orbits the planet at distances comparable to those for current Martian satellites.
\end{abstract}

I. BACKGROUND \& INTRODUCTION

Valles Marineris is the deepest trench known to exist on a terrestrial body in the solar system. There is much curiosity as to how Valles was formed. Comparing the trench to a typical water-carved structure on Earth (Figure 1) reveals the lack of the tortuous network typical for rivers and tributaries. In addition, martian surface gravitational acceleration is about $1 / 3$ that of the Earth on its surface and so it seems unlikely that water could have carved such a prominent structure on Mars. There is evidence that water or dry sand [1] ran down the sides of Valles at one point in time, but the erosion incurred from it seems clearly secondary and not related to the channel's creation [2].

$\ddagger$ current address: lowa State University (Ames, lowa).E-mail to massina4@iastate.edu
Even though it is thought that Valles could be related to a fault in the martian crust [3], it could be that the fault was not active in the channel's formation. Could it be that a grazing impact created the trench?

It is possible that a grazing impact would leave evidence which is additional to an obvious impact site. If a grazing impact was indeed responsible for carving Valles, it is likely that some of the ejecta would have been moving fast enough to leave Mars altogether or, for the proper conditions, could have ended up in orbit about Mars. There are two natural satellites for Mars: Phobos and Deimos (Figure 2). They orbit Mars in planes fairly close the planet's equator, which is also close to where Valles is located. Moreover the two moons are not spherical in shape but show a history of trauma and collision. If Valles were cut by a 

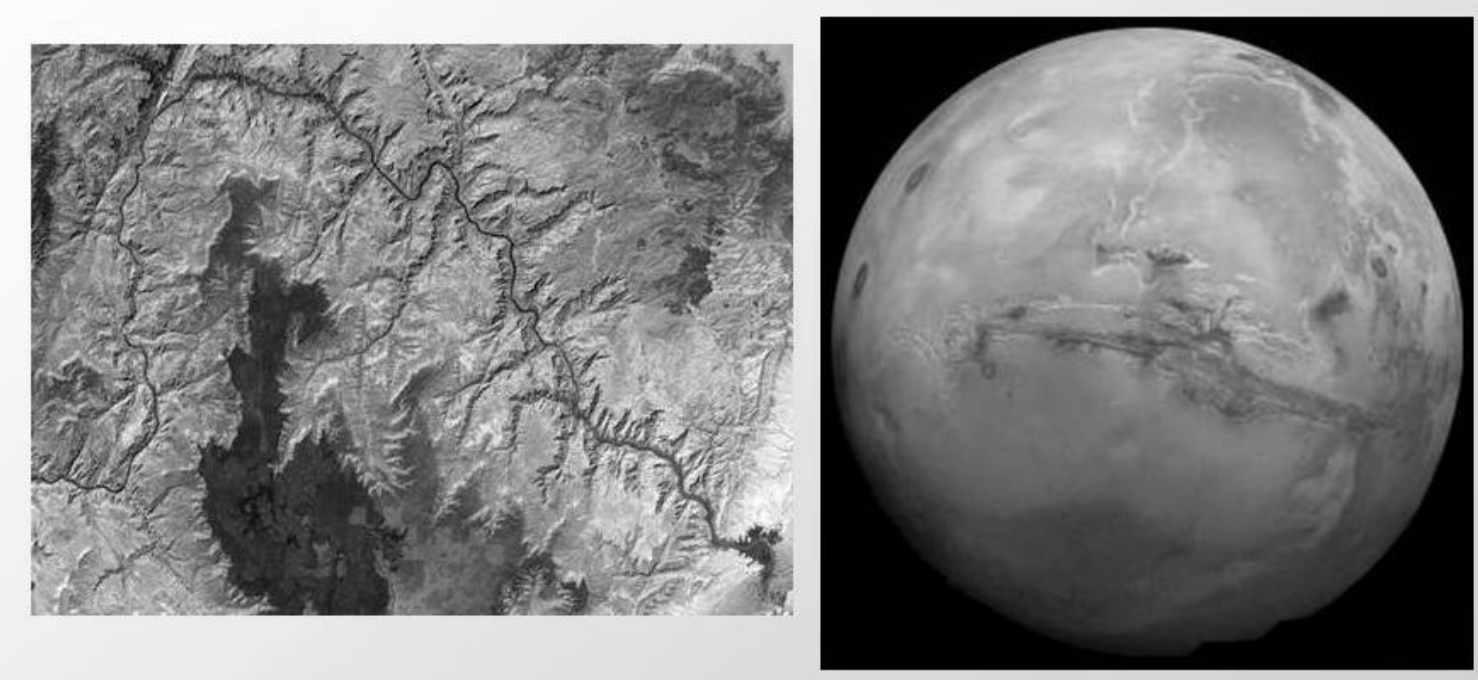

Figure1. Infrared view of Earth's Grand Canyon (left) and Valles Marineris in the visible region of the spectrum (right).

grazing impact, it seems reasonable to think that objects like Phobos and Deimos could have resulted from the debris.

The purpose of this work is to simulate grazing impacts on a terrestrial planetary surface and determine (i) if the results of a grazing impact on the surface of the planet obtained at the impact site are consistent with the general morphology of Valles and (ii) if reasonable conditions for a grazing impact can lead to debris in stable orbits about the planet and, in the case of Mars, satellites with orbits having elements similar to those of Phobos and Deimos.

\section{COMPUTATIONAL DETAILS}

The code developed consists of a combination of two different methods. The first deals with the impacting body as it travels towards the planet's surface, and is derived from classical Newtonian planetary dynamics. Since there are no collisions involved in this first phase of the simulation, it entails a two-particle center of mass attraction algorithm. Such a method is effective at economizing simulation time. This gravitational method is used until the particles enter a zone around the planet where collisions can happen and the previously mentioned center of mass dynamics are inadequate. The second algorithm that governs the collision part of the simulation is the Material Point Method.
In order to trust the results of any program, it must to be validated. This was accomplished by running simulations with the intent of reproducing kinetic and potential energy profiles of various twodimensional collisions between balls which others have simulated, as well as energy conservation in the collision of three dimensional balls. The results obtained show that the algorithm utilized for our MPM code is consistent with Newtonian physics and accurately represents a temporal mechanical sequence.

The Material Point Method (MPM) employs an algorithm developed and evaluated by Z. Chen and R. Brannon [4]. MPM is advantageous because it doesn't require a complex algorithm to simulate collisions or material failure. Instead, MPM maps the mass and momentum of groups of particles in the simulation to a background grid and utilizes the conservation equations of mass as well as that of momentum to ultimately advance the system through time. The use of the background grid also avoids entanglement associated with other contactbased algorithms.

To begin with, the initial conditions for the simulation include a collection of particles with initial positions $\left\{\vec{x}_{p}^{0}\right\}$, velocities $\left\{\vec{v}_{p}^{0}\right\}$ and boundary conditions chosen so as to reflect the important physics of the actual system as much as possible. Since the 


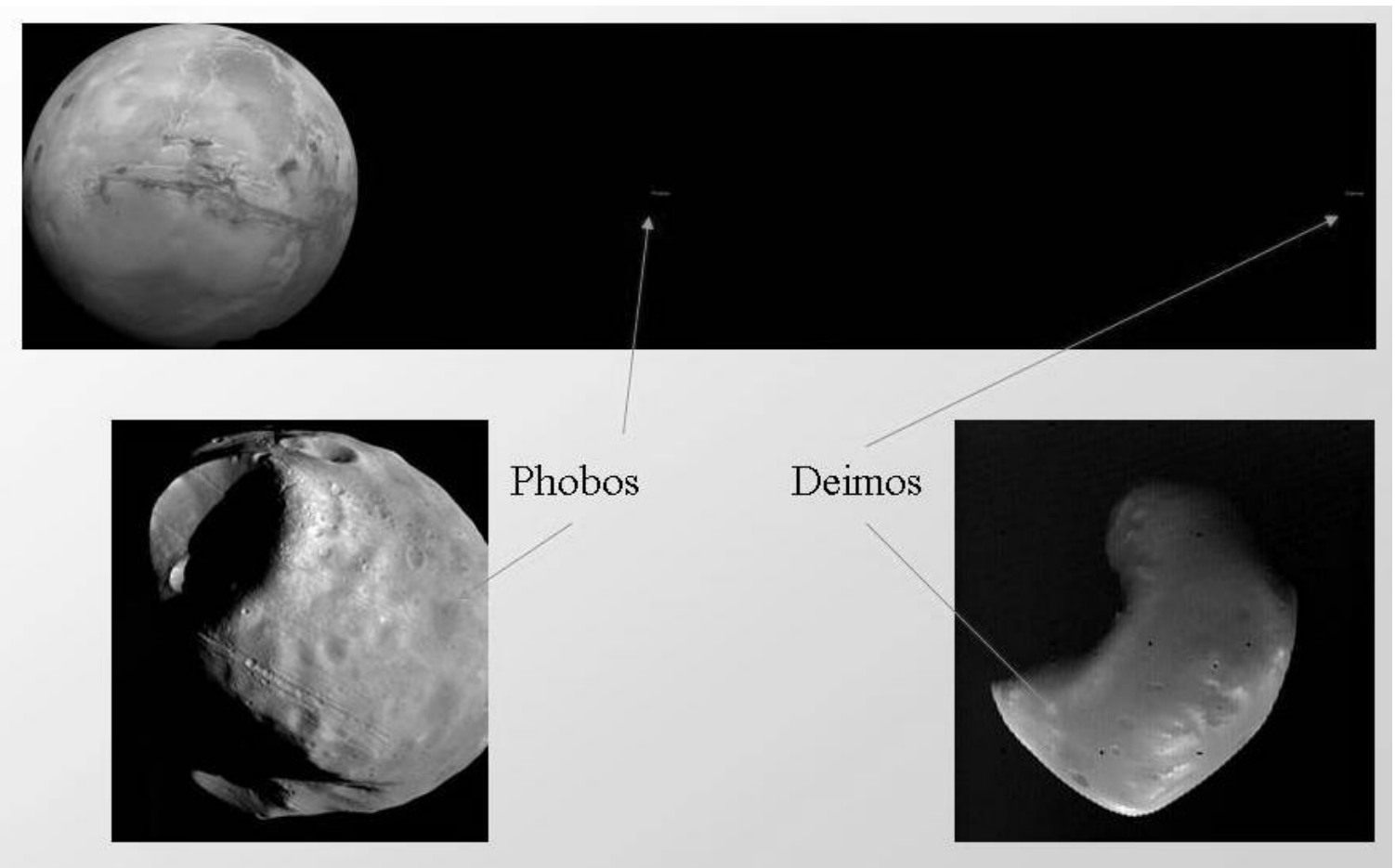

Figure 2. The two natural satellites of Mars, Phobos and Deimos, shown at their distances from Mars to scale (top) and in detail (bottom left and right).

glancing collision takes place over a very limited region of the planet, a patch of particles (the impact zone) is utilized. Next, the background grid is defined, where computational space is separated into a defined number of grid cells in which nodes are placed in each corner of each cell. The mass $M_{p}$ of each particle $p$ at time $t$ is mapped to the nodes $i$ of the grid cells based on the shape function $N_{i}\left(\vec{x}_{p}^{t}\right)$, resulting in the mass $m_{i}^{t}$ of node $i$ at time $t$ :

$$
m_{i}^{t}=\sum_{p=1}^{N p} M_{p} N_{i}\left(\overrightarrow{\mathbf{x}}_{p}^{t}\right) .
$$

The same is done for the momentum $(M v)_{p}{ }^{t}$ of the particles:

$$
(m v)=\sum_{p=1}^{N \mathrm{p}}(M \mathrm{v})_{p}^{t} N_{i}\left(x_{p}^{t}\right)
$$

The shape functions $N_{i}\left(\vec{x}_{p}^{t}\right)$ are calculated in the following way. The coordinates of the 3D box containing a particle $p$ are normalized so that they take on extreme values of 0 or 1 at vertices of the box. So if a particular box has a vertex at $\left(x_{j}, y_{j}, z_{j}\right)$ and another vertex at $\left(x_{j}+\Delta x, y_{j}+\Delta y, z_{j}+\Delta z\right)$ across its diagonal, the normalized coordinates are

$$
\begin{aligned}
& \zeta=\frac{\left(x_{p}^{t}-x_{j}\right)}{\Delta x} \\
& \eta=\frac{\left(y_{p}^{t}-y_{j}\right)}{\Delta y} \\
& \chi=\frac{\left(z_{p}^{t}-z_{j}\right)}{\Delta z}
\end{aligned}
$$

and the eight shape functions used in the particle-to-node mappings are calculated from the normalized coordinates as shown in Table 1 so that they are equal to unity for a vertex (node) where the particle is at and is zero for a given node if the particle is at any other node.

Once the mappings in equations (1) and (2) are completed then the forces at each node must be calculated. The internal force $\left(f_{i}^{t}\right)$ arises from elastic deformation of the object and is calculated as

$$
\left(\vec{f}_{i}^{t}\right)^{\text {int }}=-\sum_{p=1}^{N p} \mathbf{G}_{i}\left(\mathbf{x}_{p}^{t}\right) \bullet \mathbf{s}_{p}^{t} \frac{M_{p}}{\rho_{p}^{t}} .
$$




\begin{tabular}{|l|l|}
\hline Node Coordinates & Shape Function \\
\hline$(0,0,0)$ & $(1-\xi)(1-\eta)(1-\chi)$ \\
\hline$(1,0,0)$ & $\xi(1-\eta)(1-\chi)$ \\
\hline$(1,1,0)$ & $\xi \eta(1-\chi)$ \\
\hline$(0,1,0)$ & $(1-\xi) \eta(1-\chi)$ \\
\hline$(0,0,1)$ & $(1-\xi)(1-\eta) \chi$ \\
\hline$(1,0,1)$ & $\xi(1-\eta) \chi$ \\
\hline$(1,1,1)$ & $\xi \eta \chi$ \\
\hline$(0,1,1)$ & $(1-\xi) \eta \chi$ \\
\hline
\end{tabular}

Table 1. Expressions for the eight shape functions used to map particle properties to each of the eight vertices of the grid cube it resides in.

The external force is then calculated at each node.

$$
\vec{f}_{i}^{e x t}=\vec{c}_{i}^{t}+M_{p} \vec{g} m_{i}^{t}
$$

and then the nodal accelerations are obtained using $a_{i}^{t}=\frac{f_{i}^{t}}{m_{i}^{t}} \quad$ where the total force is given by $f_{i}^{t}=\left(f_{i}^{t}\right)^{\mathrm{int}}+\left(f_{i}^{t}\right)^{e x t}$. The node momenta are then updated in time

$$
(m v)_{i}^{t+\Delta t}=(m v)_{i}^{t}+f_{i}^{t} \Delta t
$$

and subsequently the kinematic variables associated with each particle $p$ in the simulation are updated using node-toparticle mappings:

$$
\begin{gathered}
\vec{a}_{p}^{t}=\sum_{i=1}^{N n} \frac{\vec{f}_{i}^{t}}{m_{i}^{t}} N_{i}\left(\vec{x}_{p}^{t}\right) \\
\vec{v}_{p}^{t}=\sum_{i=1}^{N n} \frac{(m \vec{v})_{i}^{t+\Delta t}}{m_{i}^{t}} N_{i}\left(\vec{x}_{p}^{t}\right) \\
\vec{x}_{p}^{t+\Delta t}=\vec{x}_{p}^{t}+\vec{v}_{p}^{t+\Delta t} \Delta t
\end{gathered}
$$

The new particle kinematic variables in eqns. (7)-(9) are mapped back onto the nodes to obtain updated nodal velocities.

Now because the simulated material(s) may have deformed in being updated, the elastic properties of the system must be taken into account. The rate of strain tensor $\vec{e}$ is now calculated from the velocity gradients: $e_{i j}=\frac{1}{2}\left(\partial_{j} x_{i}^{\prime}+\partial_{i} x_{j}^{\prime}\right)$. Here the primed variables represent velocity components and, explicitly in 3D we have

$$
\vec{e}=\left[\begin{array}{ccc}
\frac{\partial u}{\partial x} & \frac{1}{2}\left(\frac{\partial u}{\partial y}+\frac{\partial v}{\partial x}\right) & \frac{1}{2}\left(\frac{\partial u}{\partial z}+\frac{\partial w}{\partial x}\right) \\
\frac{1}{2}\left(\frac{\partial v}{\partial y}+\frac{\partial v}{\partial x}\right) & \frac{\partial v}{\partial y} & \frac{1}{2}\left(\frac{\partial v}{\partial z}+\frac{\partial w}{\partial y}\right) \\
\frac{1}{2}\left(\frac{\partial u}{\partial z}+\frac{\partial w}{\partial x}\right) & \frac{1}{2}\left(\frac{\partial u}{\partial y}+\frac{\partial u}{\partial y}\right) & \frac{\partial w}{\partial z}
\end{array}\right]
$$

Subsequently, the 3D elastic stress-strain relationship is utilized to calculate the updated stress tensor $\vec{s}^{t+\Delta t}=\vec{s}^{t}+\Delta \vec{s}$, where $\Delta s_{i j}=\sigma_{i j}=\sum_{i j k l} C_{i j k l} \varepsilon_{k l}$. Because the strain tensor in eqn. (10) is symmetric, it in general yields six nonsuperfluous elements and the stiffness tensor $C_{i j k l}$ as well as the displacement tensor $\varepsilon_{k l}$ are cast in a $6 \times 6$ format: 


$$
\left[\begin{array}{l}
\sigma_{x} \\
\sigma_{y} \\
\sigma_{z} \\
\tau_{x y} \\
\tau_{y z} \\
\tau_{z x}
\end{array}\right]=\frac{E}{(1+v)(1-2 v)}\left[\begin{array}{cccccc}
1-v & v & v & 0 & 0 & 0 \\
v & 1-v & v & 0 & 0 & 0 \\
v & v & 1-v & 0 & 0 & 0 \\
0 & 0 & 0 & \frac{1-2 v}{2} & 0 & 0 \\
0 & 0 & 0 & 0 & \frac{1-2 v}{2} & 0 \\
0 & 0 & 0 & 0 & 0 & \frac{1-2 v}{2}
\end{array}\right]\left[\begin{array}{c}
\varepsilon_{x} \\
\varepsilon_{y} \\
\varepsilon_{z} \\
\gamma_{x y} \\
\gamma_{y z} \\
\gamma_{z x}
\end{array}\right] .
$$

The algorithm then returns to mapping particle properties to the grid (eq. 1) and is repeated until a desired time limit is reached.

The impact zone was covered by anywhere from about 10000 to 120,000 small spheres (material points) placed on the surface of a larger reflecting sphere. A typical setup is shown in Figure 3, including a topsoil layer, subsurface ice layer and soil layer beneath the ice. This larger sphere represented the rest of our Martian planet not so greatly affect by the collision. The impacting body was offset and given an initial velocity such that it would hit the planet at glancing angles. Various parameters used in the simulations are presented in Table 2.

At the start of the simulation the two bodies were governed by center of mass Newtonian dynamics until the impacting body reached the collision zone. The simulation was then backed up one time step and the simulation continued governed now by MPM. Subsequently, in each step, every particle was marked to be inside or outside of the MPM grid. When a particle was inside the grid it was subjected to the MPM formalism. During and after the collision it is possible that a particle leaves the collision zone, in which case its motion returned to being governed by Newtonian planetary dynamics.

Such switching between planetary dynamics and MPM allows for better observations of ejecta to be made because, for long times after the collision there are only a few particles left near the planet but their motion needs to be examined to see if they ended up in stable orbits or not .

Current and future work involves parallelization of the code so it can handle a very large number of particles and hence

\begin{tabular}{|c|c|}
\hline Parameter & Value \\
\hline Planet Mass & 1 Martian Mass \\
\hline Planet Radius & 1 Martian Radius \\
\hline $\begin{array}{l}\text { Impacting Body } \\
\text { Mass }\end{array}$ & $\begin{array}{l}0.011 \text { Martian } \\
\text { Masses }\end{array}$ \\
\hline $\begin{array}{l}\text { Impacting Body } \\
\text { Speed }\end{array}$ & $10 \mathrm{~km} / \mathrm{sec}$ \\
\hline $\begin{array}{l}\text { Impacting Body } \\
\text { Radius }\end{array}$ & $\begin{array}{l}\text { 4.7 Phoebian } \\
\text { Radii }\end{array}$ \\
\hline $\begin{array}{l}\text { Number of Particles } \\
\text { in Impacting Body }\end{array}$ & 111 \\
\hline $\begin{array}{l}\text { Number of Particles } \\
\text { on Simulated } \\
\text { Surface }\end{array}$ & 9889 \\
\hline $\begin{array}{l}\text { Longest Simulated } \\
\text { Time }\end{array}$ & $\begin{array}{l}1 \text { Phoebian } \\
\text { month }=7.6 \mathrm{~h}\end{array}$ \\
\hline Time Step & $\begin{array}{l}5 \times 10^{-5} \text { Phoebian } \\
\text { month. = } 1.37 \\
\text { sec. }\end{array}$ \\
\hline
\end{tabular}

Table 2. Useful simulation parameters.

yield better spatial resolution and can also be run out for very long times in order to check for ejecta in stable orbits about the planet. In addition, an equation of state (EOS) relationship is being implemented in the program so that, for example, changing material properties of the ice layer upon melting due to friction and / or pressure can be incorporated in the simulation. 

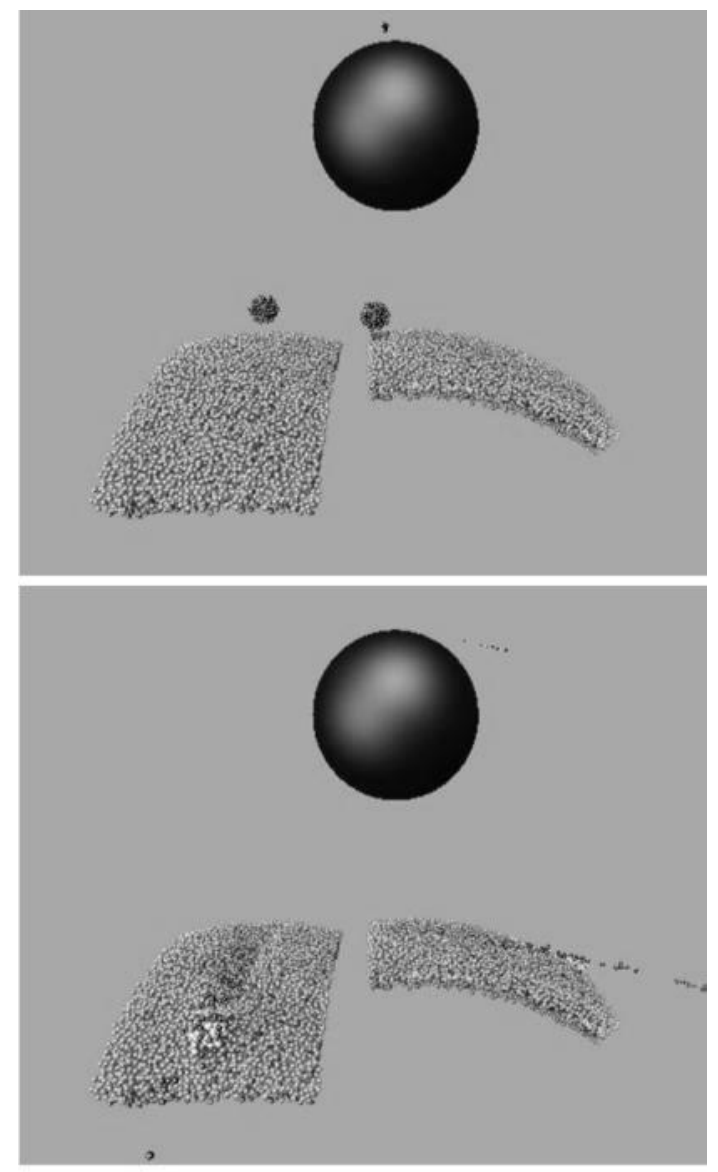

Figure 3. Initial configuration for a grazing impact simulation (top) and a typical configuration shortly after impact (bottom). The view of the whole planet (top) shows particles only governed by classical Newtonian planetary dynamics and so the impacting body disappears after impact and the surface patch is never visible. The impacting body (mottled dark/light gray); martian topsoil (gray textured sheets) becomes darker as subsurface soil is reached. A layer of ice (very light gray) separates the topsoil and subsoil.

\section{RESULTS AND DISCUSSION}

Many runs were done with the initial conditions being adjusted so that a reasonable grazing collision was obtained. Figure 3 shows the initial configuration for such a simulation. During the first stage of impact (the initial stage), colliding body elongates and breaks apart, leaving the planet's surface in a nearly straight line. Shortly after that a small amount of highspeed ejecta from the planet leaves the
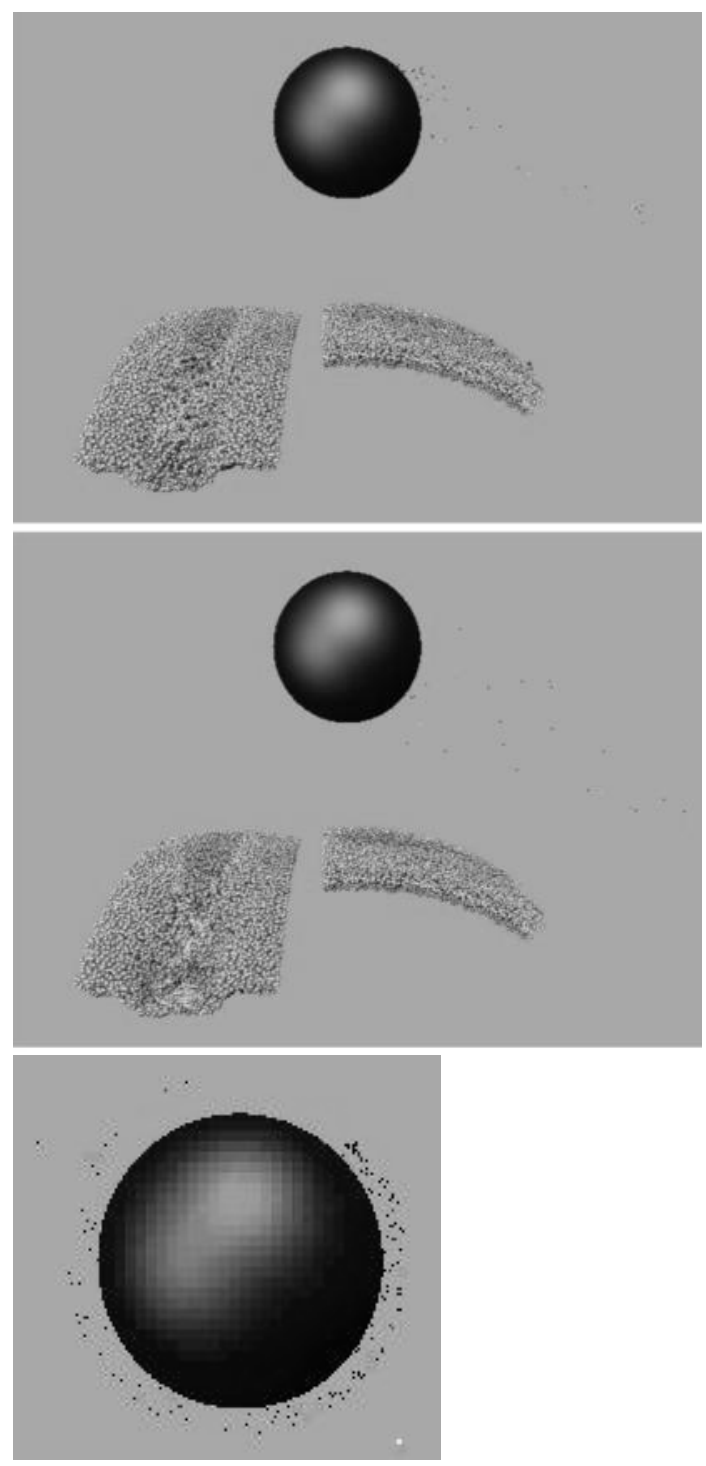

Figure 4. Typical configurations from the intermediate stage of impact (upper two panels), after the rapidly moving horizontal ejecta has left. Some simulations yielded a partial ring of debris (smaller bottom panel) during the intermediate stage of impact.

surface in a similar straight - line fashion. Next, in the intermediate impact stage slower moving ejecta from the planet as well as parts of the impacting body begin to noticeably curve in towards the surface of the planet as shown in Figure 4. In addition, on the planetary surface, the trench resulting from the impact continues to be cut out due to forward horizontal momentum delivered by the impacting body. It is noticeable in Figure 4 that the subsurface ice layer is 


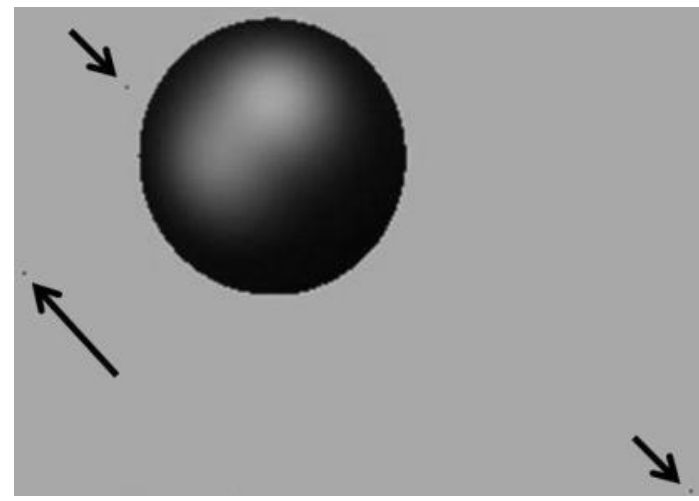

Figure 5. Some fragments of the impacting body in stable orbits about the planet in the long-term stage of the simulation.

exposed. For some initial angles and speeds of impact the intermediate stage of debris ejection involved curving of the path of debris to the extent that a temporary partial ring was formed about the planet. Such rings always extinguished, however, and there seems to be no evidence from Mars for debris from such a ring either in orbit or having fallen to the planet's surface.

In the long-term stage of the simulations there were at most two or three bodies in stable orbits about the planet, as shown in Figure 5 . The positions of the orbiting bodies have orbital elements reasonably consistent with the current martian satellites, especially Phobos.

We also examined collisions where the impacting body had a core and when the surface of impact on the planet had 120,000 particles (Figure 6). The results obtained from such simulations are similar to those previously mentioned in that the general morphology of the trench is similar to that of Valles and the ice layer in the trench is exposed. However it was noticeable that the trench had more structure due to the breakup of the core of the impacting body on the planet's surface.

\section{CONCLUSIONS}

The simulations presented here show that it is possible to have a grazing impact that cuts a trench on a planetary surface whose morphology is similar to that of Valles Marineris - narrow on the ends and wider in the middle. Considering a layer of subsurface ice it is conceivable that the grazing collision would have exposed the ice

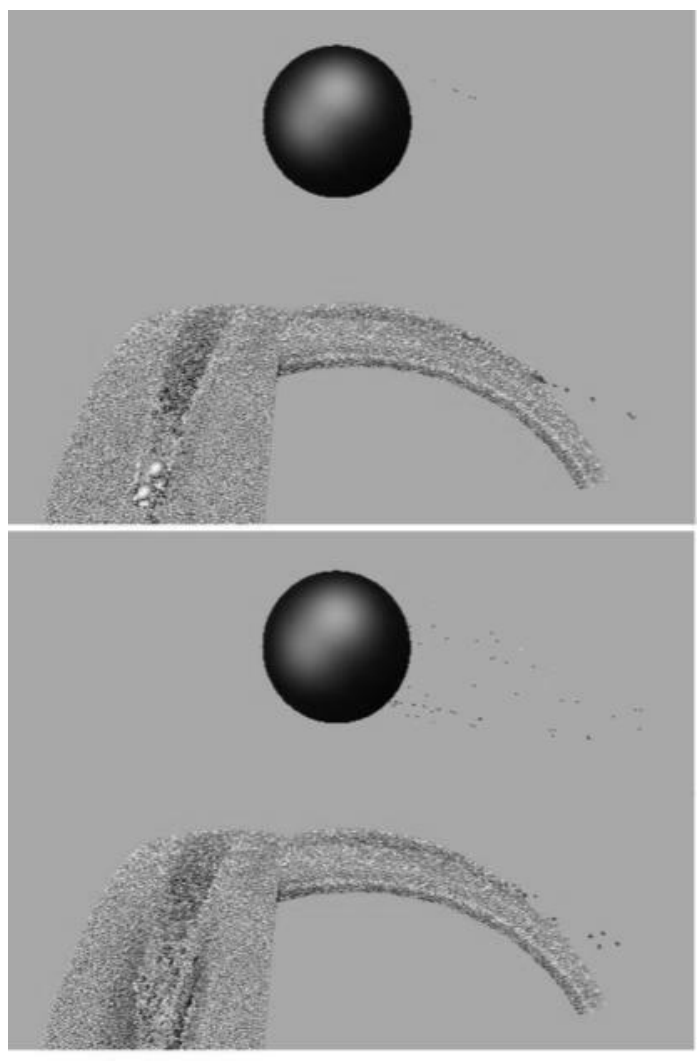

Figure 6. Configurations from simulations with 120,000 particles on the planet's impact site and a double spherical core (very light gray) for the impacting body.

layer. It also seems reasonable to think that there would be enough friction and pressure to melt the ice and spur the side wall dynamics in the trench but an equation of state (EOS) simulation, allowing phase changes in the rock and ice would be much more helpful in supporting such a notion. Moreover the simulations in this work illustrate that a grazing collision can result in debris which has stable long - term orbits about the planet reasonably consistent with those of its current satellites. Further work with larger of systems and with an EOS is certainly warranted.

\section{REFERENCES}

1. C. Quantin, , P. Allemand and C. Delacourt, Planetary and Space Science 52 (11), 1011-1022 (2004).

2. Nicolas Mangold, Cathy Quantin, Véronique Ansan, Christophe Delacourt, Pascal Allemand, Science, 305. (5680), pp. $78-81$ (July 2 2004). 
3. http://www.nasa.gov/multimedia/imageg allery/image_feature_83.html.
4. Z Chen, RM Brannon, Sandia National Laboratories (SAND2002-0482), 2002

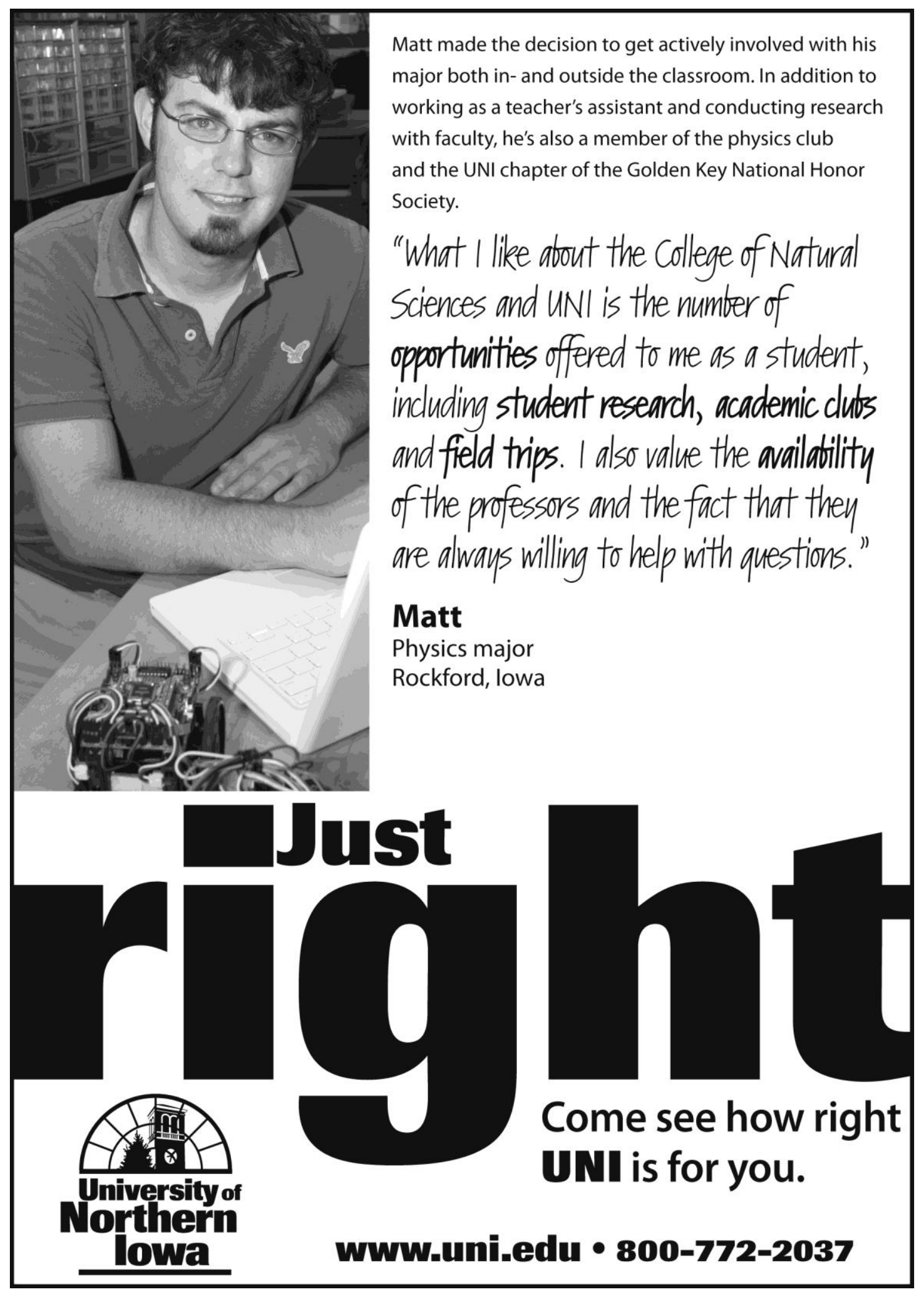

